\title{
Effects of PEGylation on the physicochemical properties and in vivo distribution of organic nanotubes
}

This article was published in the following Dove Press journal:

International Journal of Nanomedicine

12 December 2014

Number of times this article has been viewed

\section{Wuxiao Ding \\ Hiroyuki Minamikawa \\ Naohiro Kameta \\ Toshimi Shimizu \\ Mitsutoshi Masuda}

Nanosystem Research Institute, National Institute of Advanced Industrial Science and Technology (AIST), Ibaraki, Japan
Correspondence: Wuxiao Ding

Nanosystem Research Institute, National Institute of Advanced Industrial Science and Technology, Tsukuba Central 5 , Higashi I-I-I, Tsukuba 305-8565,

Ibaraki, Japan

Tel $+8|2986| 2202$

Fax +8I 298614545

Email ding-wuxiao@aist.go.jp
Abstract: Application of organic nanotubes (ONTs) into drug nanocarriers ultimately requires validation in live animals. For improving the dispersibility in biological media and in vivo distribution, the outer surface of an ONT was functionalized with polyethylene glycol (PEG) via the coassembly of an ONT-forming lipid with 5-20 mol\% of a PEG-tethered lipid analogue (PEG-lipid). Firstly, the effect of PEGylation on the psysicochemical properties of ONTs, such as morphology and dispersibility, was investigated. PEGylation of ONTs slightly reduced the average length and effectively prevented the aggregation in phosphate-buffered saline (PBS). The PEGylated ONTs even showed high thermal stability in aqueous dispersion at least up to $95^{\circ} \mathrm{C}$. Secondly, differential scanning calorimetry and powder X-ray diffraction indicated that $\sim 10 \mathrm{~mol} \%$ of PEG-lipid was completely incorporated into the ONTs, while $20 \mathrm{~mol} \%$ of PEG-lipid encountered a partial phase separation during coassembly. In the heating differential scanning calorimetry runs, the resultant PEGylated ONTs with $5 \mathrm{~mol} \%$ PEG-lipid showed no sign of phase separation up to $180^{\circ} \mathrm{C}$ under lyophilized condition, while those with $10 \mathrm{~mol} \%$ and $20 \mathrm{~mol} \%$ PEG-lipid showed some phase separation of the PEG-lipid above $120^{\circ} \mathrm{C}$. Finally, PEGylation significantly affected the tissue distribution and prolonged the persistence time in the blood in mice. Non-PEGylated ONTs was quickly cleared from the circulation after intravenous infusion and preferentially accumulated in the lung, while PEGylated ONTs was mainly trapped in the liver and could circulate in the blood up to 24 hours. This study provided valuable information of physicochemical properties and the in vivo distribution behavior of PEGylated ONTs for their potential application into drug nanocarriers.

Keywords: nanostructure, dispersibility, distribution

\section{Introduction}

One-dimensional nanostructures have recently emerged as promising drug nanocarriers at both cell and small animal levels. Carbon nanotube and polymeric nanoparticles with high aspect ratio morphologies enjoyed appreciable advantages in the internalization mechanism and rate into cancer cells, ${ }^{1-3}$ while flexible polymer rod-like micelles showed much longer blood persistence than spherical counterparts in mice and rats. ${ }^{4} \mathrm{~A}$ solid form of lipid self-assemblies, organic nanotubes (ONTs), have also been reported for their applications in the nanomedicine field. ${ }^{5}$ The large ONT from diacetylenic lipid 1,2-bis(tricosa10,12-diynoyl)-sn-3-phosphocholine possessed a diameter of 500-1,000 nm and provided the sustained release not only for macromolecular proteins such as transforming growth factor- $\beta$, but also for small drugs. ${ }^{6-10}$ Further functionalization of ONTs offers a selective and efficient drug loading, and controlled release. Some recent studies have demonstrated that incorporating charge and hydrophobicity onto the nanotube inner surfaces leads to encouraging applications for in vitro drug delivery. ${ }^{11-18}$ 
A couple of studies of drug-loaded ONTs in animals have also been reported for oral gene delivery, ${ }^{19}$ intravitreal delivery of anticancer drugs, ${ }^{20}$ and intravenous delivery of irinotecan. ${ }^{21}$ An ONT with a length of $2 \mu$ m outer diameter of 70-90 nm was found to accumulate specifically in the lung and be quickly cleared from blood circulation. ${ }^{21}$ Although the observed size of this ONT is less than $100 \mathrm{~nm}$, the carboxyl group-covered hydrophobic surface led this ONT to form large bundles and be poorly dispersed in water. Further, this ONT was biologically unstable because the ONT was decomposed into lipid at a $\mathrm{pH}$ of 7.4. In contrast to those poorly dispersed ONTs, ${ }^{21,22}$ we have successfully developed highly water-dispersed and biologically stable ONTs with an outer diameter less than $20 \mathrm{~nm} \cdot{ }^{15,16,23}$ In our in vitro studies, these ONTs are effective as nanocarriers in terms of high loading for anticancer drugs and proteins and controlled release property, but in vivo validation is indispensable to advance the ONTs into potential drug nanocarriers. We expect that these ONTs having smaller size, higher dispersibility, and higher stability may improve their biodistribution and biological effect.

The modification of polyethylene glycol (PEG) on the surface of nanocarriers, called "PEGylation," has been widely used to prevent liposomes, ${ }^{24}$ polymeric nanoparticles,${ }^{25}$ and carbon nanotube ${ }^{26}$ from premature clearance during circulation in the blood, since the PEGylation improves dispersibility and reduces the uptake by mononuclear phagocyte system. PEGylation of ONT has also been previously reported as a dispersant in preventing the aggregation and in vitro transfection of the ONT-plasmid DNA complex. ${ }^{13}$ In this work, we further modified the mother ONT (self-assembly of ONT-forming lipid alone) with dual functionalities by a coassembly approach in order to reveal the effect of PEGylation on in vivo distribution. PEG (MW 2000) chain was selectively anchored on the outer surface to provide good dispersibility in physiological condition, and tetraazacyclododecane tetraacetic acid (DOTA) groups on the inner surface for complexation with gadolinium ion for the detection of ONTs (Figure 1). The physicochemical properties of ONTs, such as morphology, dispersibility, and thermal stability were investigated. Finally, the in vivo distribution of the PEGylated ONTs was evaluated by measuring the gadolinium ion, and the existence of PEGylated ONTs in the plasma was also directly visualized by scanning transmission electron microscopic (STEM) observation.

\section{Experimental methods}

\section{Materials}

4-(4,6-Dimethoxy-1,3,5-triazin-2-yl)-4-methylmorpholinium chloride (DMT-MM), ethylenediamine, Tri-tert-butyl
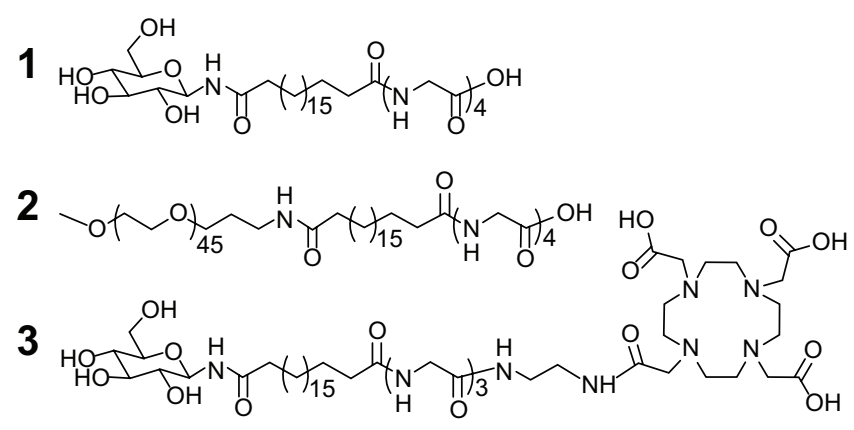

Figure I ONT-forming lipid I, PEG-lipid 2, and DOTA-lipid 3 for the construction of ONTs.

Notes: These lipids possess common octadecane hydrophobic spacer and oligoglycine moiety for the coassembly into ONTs via hydrophobic interaction and hydrogen bonds.

Abbreviations: DOTA, tetraazacyclododecane tetraacetic acid; ONT, organic nanotube; PEG, polyethylene glycol.

1,4,7,10-tetraazacyclododecane-1,4,7,10-tetraacetate (triBoc-DOTA), and trifluoroacetic acid were purchased from the Tokyo Chemical Industry Co, Ltd (Japan), All organic solvents used for the synthesis and coassembly were of analytical grade.

\section{Synthesis of PEG-lipid 2 and DOTA-lipid 3}

PEG-tethered lipid analogue (PEG-lipid) 2 was synthesized by the coupling of mono-PEGylated eicosanedioic acid (MW of PEG: 2000 ${ }^{13}$ with glycyl-glycyl-glycyl-glycine methyl ester $^{15}$ using DMT-MM as a coupling agent (Figure 2). After 4 hours' reaction at room temperature, methanol was removed and the residue was redispersed in water for dialysis (MWCO 3,500). The water dispersion was hydrolyzed to remove the methyl group by sodium hydroxide solution, followed by $\mathrm{pH}$ adjustment to acidic condition and dialysis to remove sodium chloride. The final dispersion was freeze-dried to get PEG-lipid 2. ${ }^{1} \mathrm{H}-\mathrm{NMR}$ (400 MHz, DMSO- $\left.d_{6}\right): \delta 7.74-8.02\left(\mathrm{~m}, 4.8 \mathrm{H},-\mathrm{CONHCH}_{2}-\right), 3.75$ and 3.83 (d, $8 \mathrm{H}$; - $\left.\mathrm{NHCH}_{2} \mathrm{CO}-\right), 3.42\left(\mathrm{~m}, 208 \mathrm{H},-\mathrm{OCH}_{2} \mathrm{CH}_{2} \mathrm{O}-\right)$, $3.18\left(\mathrm{~S}, 3 \mathrm{H},-\mathrm{OCH}_{3}\right), 2.98\left(\mathrm{dd}, 2 \mathrm{H},-\mathrm{CONHCH}_{2} \mathrm{CH}_{2}-\right), 1.98(\mathrm{t}, 4$ $\mathrm{H},-\mathrm{CH}_{2} \mathrm{CH}_{2} \mathrm{CONH}-$ ), 1.52 (dd, $2 \mathrm{H}$, - $\mathrm{CONHCH}_{2} \mathrm{CH}_{2} \mathrm{CH}_{2} \mathrm{O}-$ ), $1.41\left(\mathrm{~m}, 4 \mathrm{H},-\mathrm{NHCOCH}_{2} \mathrm{CH}_{2}-\right), 1.16\left(\mathrm{~m}, 28 \mathrm{H},-\mathrm{CH}_{2}^{-}\right)$.

DOTA-lipid 3: 19-( $N$ - $\beta$-D-glucopyranosyl carbamoyl) nonadecanoyl-glycyl-glycyl-glycyl-ethyl ester was synthesized in a similar process as previously reported. ${ }^{15}$ It was aminolyzed with excess ethylenediamine in dimethyl sulfoxide (DMSO) at $80^{\circ} \mathrm{C}$. Addition of methanol gave a precipitation of $19-(N-\beta$-D-glucopyranosyl carbamoyl) nonadecanoyl-glycyl-glycyl-glycyl-ethylamine. Then it was conjugated with triBoc-DOTA using DMT-MM as a coupling agent in DMSO. The DMSO dispersion was freeze-dried and methanol was added to remove excess 
A

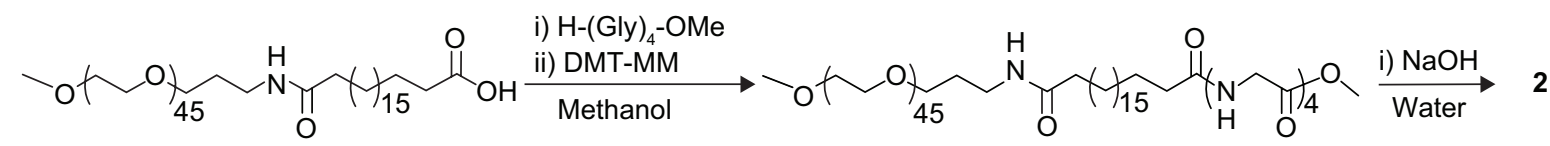

B
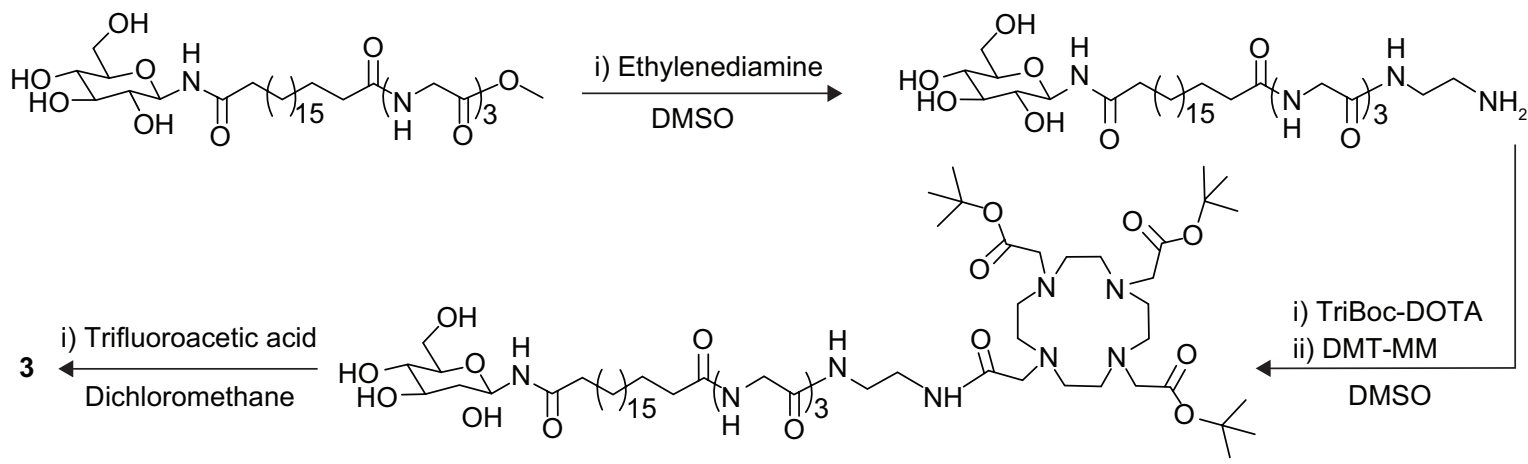

Figure 2 Synthesis of (A) PEG-lipid 2 and (B) DOTA-lipid 3.

Abbreviations: DMSO, dimethyl sulfoxide; DOTA, tetraazacyclododecane tetraacetic acid; PEG, polyethylene glycol.

unreacted 19-( $N$ - $\beta$-D-glucopyranosyl carbamoyl) nonadecanoyl-glycyl-glycyl-glycyl-ethylamine by filtration. The filtrate methanol solution was evaporated and water was added to precipitate triBoc-DOTA conjugated lipid. The precipitated solid was filtrated, dried under vacuum, and dissolved in dichloromethane/trifluoroacetic acid to remove the protecting Boc groups. The solution was evaporated to obtain DOTA-lipid 3. ${ }^{1} \mathrm{H}-\mathrm{NMR}\left(400 \mathrm{MHz}, \mathrm{DMSO}-d_{6}\right)$ : $\delta$ 8.04-8.4 (m, $\left.4.9 \mathrm{H},-\mathrm{CONHCH}_{2}-\right), 4.64(\mathrm{dd}, 0.9 \mathrm{H} ;-\mathrm{OCHN}-$ $\mathrm{HCO}-$ ), 3.75 and 3.83 (m, $13 \mathrm{H}$; - $\mathrm{NHCH}_{2} \mathrm{CO}-,-\mathrm{NCH}_{2} \mathrm{CO}-$ ), 3.1-3.3 (m, $28 \mathrm{H}$; $\mathrm{H}$ of glucose, $\left.-\mathrm{NHCH}_{2} \mathrm{CH}_{2} \mathrm{NH}-\right), 2.02$ (t, $\left.4 \mathrm{H},-\mathrm{CH}_{2} \mathrm{CH}_{2} \mathrm{CONH}-\right), 1.41$ (m, $4 \mathrm{H},-\mathrm{NHCOCH}_{2} \mathrm{CH}_{2}^{-}$), $1.16\left(\mathrm{~m}, 28 \mathrm{H},-\mathrm{CH}_{2}^{-}\right)$.

\section{Construction of PEGylated ONT and PEGylated DOTA-ONT}

PEGylated ONTs were prepared by the coassembly of ONT-forming lipid 1 and PEG-lipid 2 (Figure 1). Sodium salts of $\mathbf{1}$ and $\mathbf{2}$ (with $0,5,10$, and $20 \mathrm{~mol} \%$ of 2) were first dissolved in 2-propanol- $\mathrm{H}_{2} \mathrm{O}$ mixed solvent $(1 / 2, \mathrm{v} / \mathrm{v})$ under mild alkaline conditions ( $\mathrm{pH} \sim 8.5$ ), and the solution was neutralized with dilute hydrochloric acid to a $\mathrm{pH}$ around 4.0 to form ONTs. Correspondingly, they were named as mother ONT, 5\% PEG-ONT, 10\% PEG-ONT, and 20\% PEG-ONT, respectively. 2-propanol was removed by evaporation, and the concentration of $\mathbf{1}$ was adjusted to $5 \mathrm{mg} / \mathrm{mL}$. Morphologies of ONTs were confirmed by the STEM observation, and length distribution was calculated by counting $\sim 100$ nanotubes in the STEM images. Selfassembly of $\mathbf{2}$ was prepared by the similar $\mathrm{pH}$ adjustment in water. The $\mathrm{NaCl}$ in the mother ONT dispersion was removed by membrane filtration and the mother ONT was redispersed in water.

DOTA-ONT was prepared by the same method using 1 and 3, while PEGylated DOTA-ONT was formulated with $\mathbf{1}, \mathbf{2}$, and $\mathbf{3}$. Self-assembly of $\mathbf{3}$ was prepared by the similar $\mathrm{pH}$ adjustment in water. Ten mol\% 2 and 10 mol\% 3 were mixed with lipid $\mathbf{1}$. Any free lipid $\mathbf{3}$ was removed by dialysis (MWCO 12,000) since it is soluble in water under $\mathrm{pH}$ 4.0. The final nanotube dispersion was concentrated to $5 \mathrm{mg} / \mathrm{mL}$ of $\mathbf{1}$, and the $\mathrm{pH}$ was adjusted within $6.5 \sim 7$ for the complexation with gadolinium (III) acetate (0.9 equivalent of 3 ) on the ONT inner surfaces. The gadolinium (III) concentration was about $0.584 \mu \mathrm{mol} / \mathrm{mL}$ as measured by inductively coupled plasma-atomic emission spectroscopy (ICP-AES, Horiba Ultima). Dispersibility and size of those ONTs with DOTA ligands were essentially the same with corresponding counterparts without DOTA ligands. For brevity in the following text, DOTA-ONT and PEGylated DOTA-ONT refer to the gadolinium-labeled ONTs.

\section{Differential scanning calorimetry and powder X-ray diffraction analysis of ONTs and self-assembly of 2}

About $20 \mathrm{mg}$ of freeze-dried ONTs or self-assembly of $\mathbf{2}$ was hermetically sealed into a silver capsule and the differential scanning calorimetry (DSC) curves were recorded at a heating rate of $2^{\circ} \mathrm{C} /$ minute. The $\mathrm{X}$-ray diffraction (XRD) patterns of $10 \%$ PEG-ONT and self-assembly of 2 were measured with a Rigaku Type 4037 diffractometer using graded $d$-space elliptical side-by-side multilayer optics, monochromated $\mathrm{Cu}-\mathrm{K} \alpha$ radiation $(40 \mathrm{kV}, 30 \mathrm{~mA})$, and an imaging plate 
(R-Axis IV). The exposure time was 30 minutes with a $150 \mathrm{~nm}$ sample-to-camera length. The temperature of XRD samples was controlled to room temperature and $170^{\circ} \mathrm{C}$ with a Mettler Toledo FP92 hotstage.

\section{Dispersion experiment}

The size change of ONTs in deionized water and phosphatebuffered saline (PBS, pH 7.4) was evaluated by the dynamic light scattering (DLS) method (Malvern Zetasizer Nano). To study the thermal stability of ONT dispersions, $1 \mathrm{~mL}$ of $10 \%$ PEG-ONT dispersion was put into two glass vials; one was kept at room temperature, and the other was treated in an oil bath $\left(95^{\circ} \mathrm{C}\right)$ for 5 minutes. After cooling to room temperature, $10 \times \operatorname{PBS}(0.1 \mathrm{~mL})$ was added into the dispersion samples and the turbidity was recorded on a Hitachi U-3900H UV-Vis spectrometer. The mother ONT was used as a negative control.

\section{In vivo distribution of DOTA-ONT and PEGylated DOTA-ONT}

The animal experiments were performed with the approval of the life science division of AIST. Each group used four to five mice with an average body weight of $27 \mathrm{~g}$. Injected dose of gadolinium is about $4.33 \mu \mathrm{mol} / \mathrm{kg}$ for both ONTs. Since the quick intravenous injection of DOTA-ONT $(200 \mu \mathrm{L}$, $5 \mathrm{mg} / \mathrm{mL}$ of the 1) resulted in the instant death of the mice, it was diluted into $1 \mathrm{mg} / \mathrm{mL}$ for slow intravenous infusion into mice. Nevertheless, infusion of DOTA-ONT still resulted in a poor physical condition of mice, and many died after 1 hour. Therefore, the distribution of DOTA-ONT was only investigated at 1 hour. The PEGylated DOTA-ONT $(200 \mu \mathrm{L}$, $5 \mathrm{mg} / \mathrm{mL}$ of the 1) was quickly injected into mice and distribution was studied until 24 hours. Blood samples $(\sim 0.8 \mathrm{~mL})$ were taken from the portal vein and the mice were sacrificed at 1, 6, and 24 hours after injection. Lung, liver, kidney, and spleen were removed, rinsed in saline, and weighed for digestion. Blood samples $(0.5 \mathrm{~mL})$ were digested by $2 \mathrm{~mL}$ of $\mathrm{HNO}_{3}$, and the mixture was evaporated to dryness at $100^{\circ} \mathrm{C}$ with an electric hot plate. Finally, $10 \mathrm{~mL}$ of $\mathrm{HNO}_{3}$ solution (1 M) was added to dissolve the solid. These whole organs were cut into small pieces and digested by mixed acid of $\mathrm{HNO}_{3}-\mathrm{HClO}_{4}(4: 1, \mathrm{v}: \mathrm{v})$ for 48 hours at room temperature, and then evaporated to dryness at $100^{\circ} \mathrm{C}$ on an electric hot plate. Finally, $10 \mathrm{~mL}$ of $\mathrm{HNO}_{3}$ solution $(1 \mathrm{M})$ was added to dissolve the solid. These solutions were allowed to settle at room temperature for 2 days, and the gadolinium concentration in the clear supernatant was measured by ICP-AES (detected at $342.246 \mathrm{~nm}$ ). The blood volume was set as
$0.0778 \mathrm{~mL} / \mathrm{g}$ body weight to calculate the whole amount of gadolinium in the blood. ${ }^{27}$

For the direct visualization of PEGylated DOTA-ONT in the plasma, blood samples were collected at 5 minutes, 1 hour, 6 hours, and 24 hours and were centrifuged at $3,500 \mathrm{rpm}$ to get the plasma. Ten microliters of plasma samples were diluted into $1 \mathrm{~mL}$ water containing $10 \mu \mathrm{L}$ of staining agent $2 \%$ phosphotungstate solution. Two microliters of the diluted samples were uniformly dried on a carbon grid for STEM observation. The ONT numbers in the typical STEM images partially reflected the ONT concentration in the blood.

\section{Results and discussion Morphologies of ONTs and self- assemblies from 2 and 3}

The mother ONT and PEGylated ONTs had uniform tubular morphologies and similar outer diameters of 16-18 nm except the 20\% PEG-ONT (Figure 3A-D). By contrast, lipid 2 self-assembled into irregular nanostructures (Figure $3 \mathrm{E}$ ). Such irregular nanostructures were essentially not found in the STEM images of PEGylated ONTs. The mother ONT, 5\% PEG-ONT, and 10\% PEG-ONT showed fine tubular morphologies with regular open ends (Figure 2A-C), while $20 \%$ PEG-ONT produced many broken nanotube fragments and a minor amount of large tubes (40 $\mathrm{nm}$ outer diameter, arrow marks in Figure 3D). The STEM result suggested that, at $20 \mathrm{~mol} \%$ of PEG-lipid 2, phase separation of $\mathbf{2}$ or coexistence of other assemblies might have occurred during the coassembly process. The DOTA-lipid 3 was designed to selectively anchor on the inner surfaces of DOTA-ONT and PEGylated DOTA-ONT. At pH 4.5, DOTA-lipid 3 selfassembled into fine nanofibers with a size between 3.0 and $6.0 \mathrm{~nm}$ (Figure 3F and Figure S1). Ten mol\% of 3 seemed to have coassembled into DOTA-ONT and PEGylated DOTAONT, since they showed fine tubular morphologies and such nanofibers were not found in the STEM images (Figure 3G and $\mathrm{H}$ ). The dispersibility and size of DOTA-modified ONTs were essentially the same with corresponding counterparts without DOTA ligands.

PEGylation affected the length distribution of ONTs. The mother ONT showed a major length distribution between 400 and $600 \mathrm{~nm}$ (Figure 4). The ONT modified with $5 \mathrm{~mol} \%$ of 2 did not show a large difference in length distribution from the mother ONT, while those with $10 \mathrm{~mol} \%$ and $20 \mathrm{~mol} \%$ of 2 became shorter in the length range of 200 to $400 \mathrm{~nm}$. These effects on morphology and length suggested that the bulky and hydrophilic PEG chain might perturb the intermolecular 

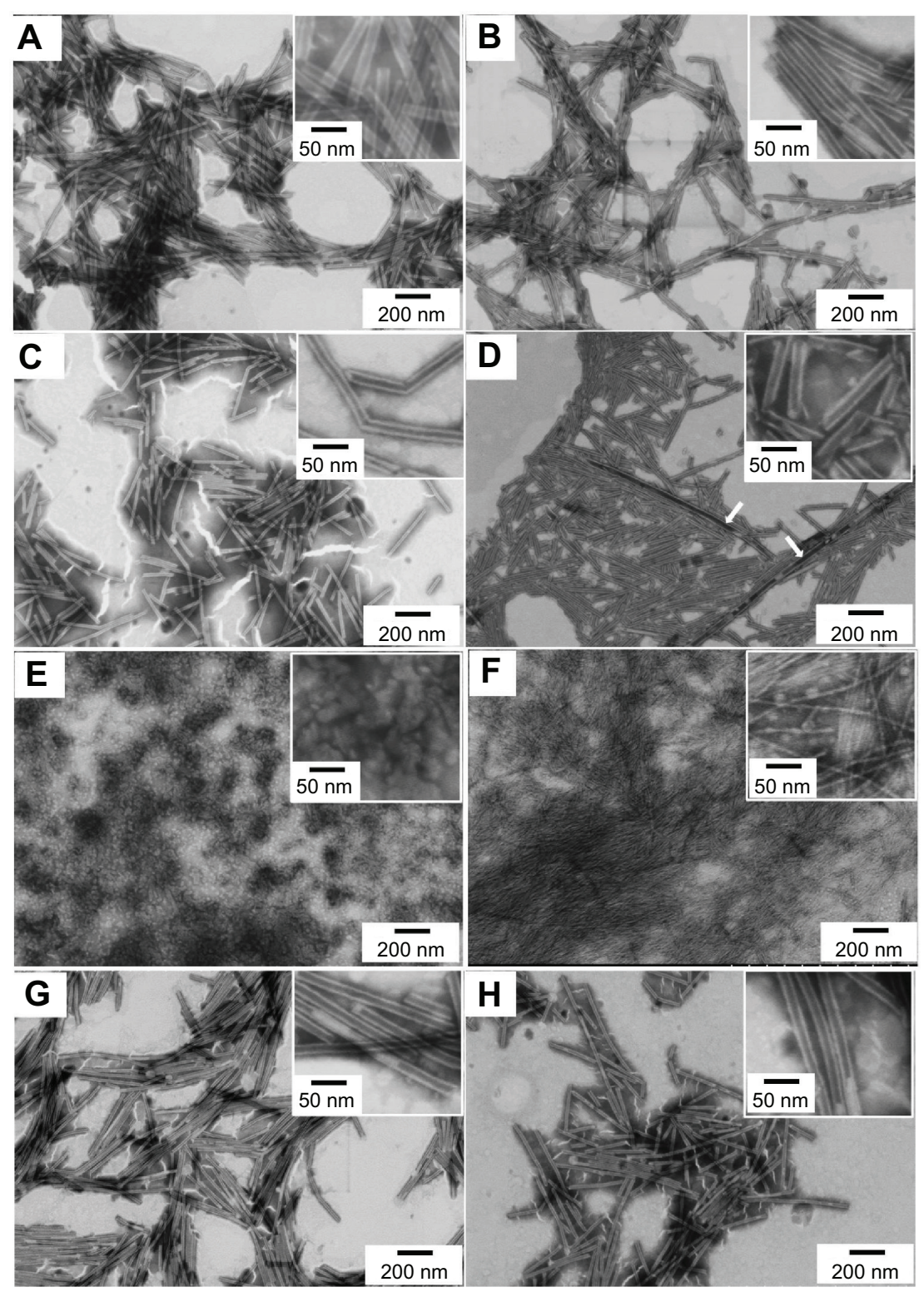

Figure 3 Morphologies of ONTs and self-assemblies of 2 and $\mathbf{3}$ based on STEM observation.

Notes: (A) Mother ONT, (B) 5\% PEG-ONT, (C) 10\% PEG-ONT, (D) 20\% PEG-ONT, (E) Self-assembly of 2, (F) Self-assembly of 3, (G) DOTA-ONT, and (H) PEGylated DOTA-ONT. The samples were negatively stained with $2 \%$ phosphotungstate, allowing the cylindrical nanochannels of the ONTs to be visualized because they were darker than the outer walls of the ONTs.

Abbreviations: DOTA, tetraazacyclododecane tetraacetic acid; ONT, organic nanotube; PEG, polyethylene glycol; STEM, scanning transmission electron microscopy.

association or the molecular arrangement between lipids at a high fraction of $\mathbf{2}$.

\section{Dispersibility and thermal stability of ONTs in PBS buffer}

DLS measurement revealed the PEGylation dramatically improves the dispersibility of ONTs in PBS buffer. The dispersibility of ONTs in deionized water and PBS were investigated by DLS measurement at room temperature. Although the measured mean size (Figure 5) did not precisely reflect the length of ONTs (Figure 4), it could definitely reflect the aggregated/dispersed status. Figure 5 clearly showed that the mother ONT aggregated in PBS (pH 7.4) with the increase of average size from $180 \mathrm{~nm}$ to more than $10 \mu \mathrm{m}$, while the PEGylated ONTs stayed between 120 and $160 \mathrm{~nm}$ in PBS. Good dispersibility of mother ONT in water may be attributable to the repulsive surface charge arising from an excess adsorption of hydroxyl ions $\left(\mathrm{OH}^{-}\right)$onto the surface as reported previously. ${ }^{28}$ In the buffer solutions, the increased ions may shield the surface charge, and cause the aggregation of the nanotubes. On the other hand, the PEGylated ONTs still had the steric repulsion of the PEG chain to 


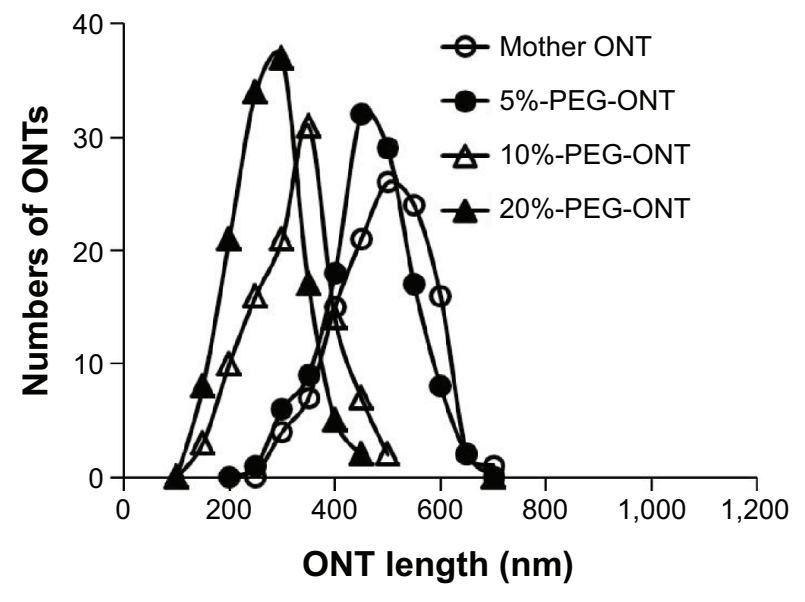

Figure 4 Length distribution of mother ONTs and PEGylated ONTs. Abbreviations: ONT, organic nanotube; PEG, polyethylene glycol.

keep the nanotubes well dispersed even in the buffers. This dispersibility experiment also suggested that $\mathbf{2}$ was effectively anchored onto the outer surface of PEGylated ONTs. Similarly to the phenomenon in PBS, the mother ONT also formed agglomeration in mouse plasma (Figure S2), while the PEGylated ONTs effectively prevented the agglomeration in both PBS and plasma.

Further, $10 \%$ PEG-ONT was stable enough to retain the PEG chain on its outer surface even at $95^{\circ} \mathrm{C}$ in water. Dissociation of PEG-conjugated lipid from self-assembled vehicles was commonly observed over the gel-liquid crystal phase transition temperatures or under dilute conditions. ${ }^{29}$ Toward practical applications, the retention of PEG-lipid on ONTs is an important physicochemical property regarding the storage and heat sterilization of PEGylated ONTs. Ten percent

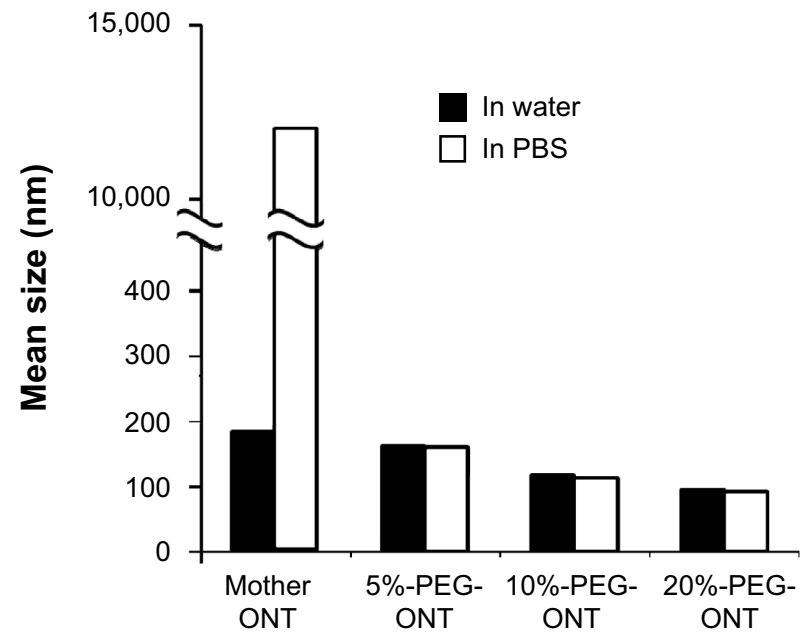

Figure $\mathbf{5}$ Mean particle size of ONTs in water and PBS as measured by DLS. Abbreviations: DLS, dynamic light scattering; ONT, organic nanotube; PBS, phosphate-buffered saline; PEG, polyethylene glycol.
PEG-ONT dispersion was selected because of its high fraction of $\mathbf{2}$ and fine tubular morphology. The transmittance of the heat-treated $10 \%$ PEG-ONT dispersion in PBS buffer was exactly the same as the nontreated one (Figure 6). Since the complete dissociation of $\mathbf{2}$ from $10 \%$ PEG-ONT would result in an aggregation in PBS, this result suggested that the coassembled 2 was stably anchored on ONT even at $95^{\circ} \mathrm{C}$ in aqueous dispersion, offering a possibility for the long-term storage and heat sterilization of the PEGylated ONTs. At a lower fraction of 2, 5\% PEG-ONT was most likely to have the same thermal stability.

\section{DSC and XRD analysis}

The coassembled status and maximum amount of $\mathbf{2}$ in ONTs were analyzed by DSC analysis, which has been widely employed for studying the phase behavior of liposome formulations. ${ }^{30,31}$ The DSC results showed that up to $10 \mathrm{~mol} \%$ of 2 was completely coassembled into the ONTs without phase separation. In the DSC measurement (Figure 7, Table S1), the mother ONT showed good thermal stability since no endothermic or exothermic peaks were found below $180^{\circ} \mathrm{C}$. The high thermal stability of ONT may be attributed to the thermally stable solid structure due to the strong intermolecular interactions (hydrogen bonding and hydrophobic interaction). By contrast, selfassembly of $\mathbf{2}$ showed two pronounced peaks with peak values of $53^{\circ} \mathrm{C}$ and $103^{\circ} \mathrm{C}$, of which the endothermic peak at $53^{\circ} \mathrm{C}$ was associated with the melting of PEG chain..$^{32}$

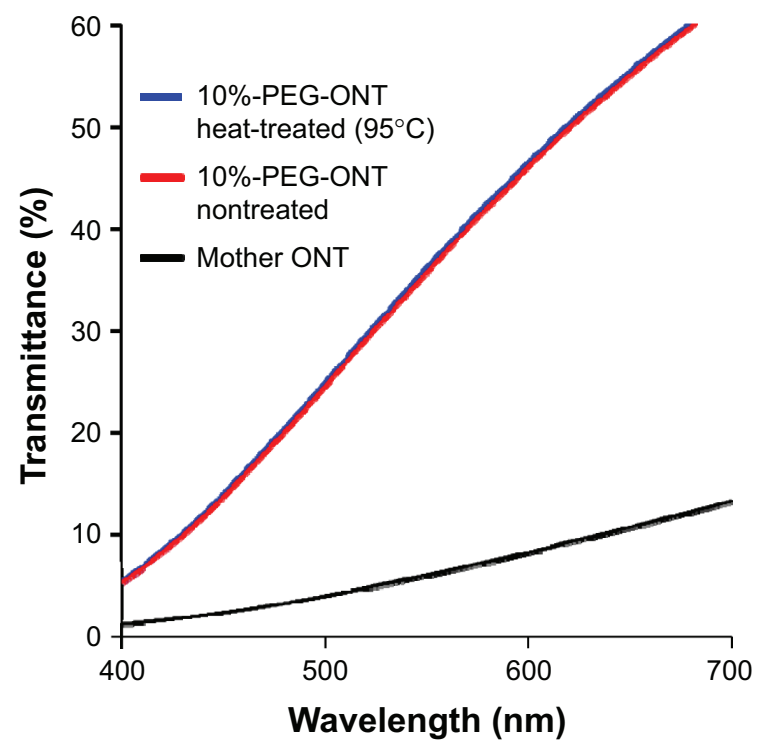

Figure 6 Transmittance of 10\% PEG-ONT and mother ONT dispersions in PBS buffer at room temperature.

Abbreviations: ONT, organic nanotubes; PBS, phosphate-buffered saline; PEG, polyethylene glycol. 


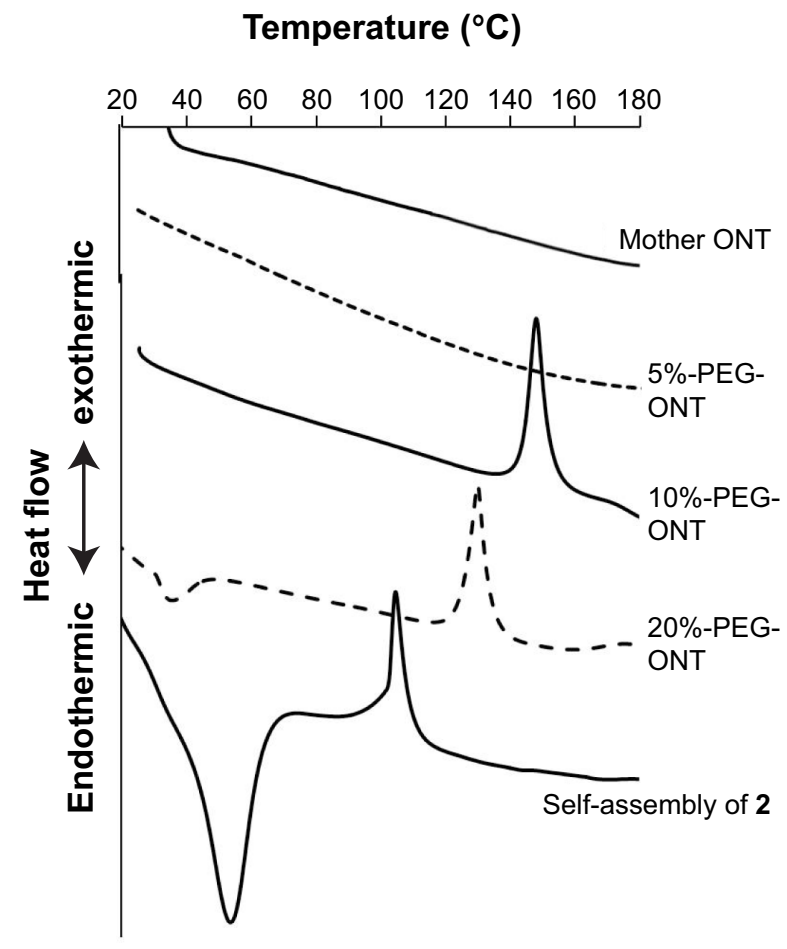

Figure 7 DSC curves of ONTs and self-assembly of 2. Abbreviations: ONT, organic nanotube; PEG, polyethylene glycol.

Five percent and 10\% PEG-ONT did not show such endothermic peaks at the lower temperature region, while $20 \%$ PEG-ONT showed a small but evident endothermic peak at $37^{\circ} \mathrm{C}$ corresponding to the melting of PEG chain. This result suggested that, during the coassembly process, phase separation of 2 from ONTs did not occur at $5 \mathrm{~mol} \%$ and $10 \mathrm{~mol} \%$ of PEG-lipid 2, while some phase separation occurred at a high PEG-lipid 2 content ( $20 \mathrm{~mol} \%)$ and might contribute to the formation of larger tubes (indicated by the arrows in Figure 3D). It was reported that the maximum composition of $\mathrm{PEG}_{2000}$-phospholipid incorporated into liposomes was about $7.5 \mathrm{~mol} \%{ }^{30,33}$

Pronounced exothermic phase transition was found for $10 \%$ PEG-ONT at $148^{\circ} \mathrm{C}$ and $20 \%$ PEG-ONT at $129^{\circ} \mathrm{C}$. They were plausibly due to the heat-induced phase separation, such as detachment of $\mathbf{2}$ from ONTs for the following reasons: (1) The polarized optical microscopy indicated that the phase transition of $10 \%$ PEG-ONT was a solid-to-solid type, while that of self-assembly of $\mathbf{2}$ was a solid-to-liquid one (Figure S3). (2) The transition occurred only on the first heating (Figure S4). (3) After the transition, 10\% PEG-ONT lost its high dispersive nature in PBS buffer but retained tubular structure (Figure S5). (4) The sharp diffraction peak of $\mathrm{PEG}_{2000}$ chain in $10 \%$ PEG-ONT almost disappeared after heating as described in the coming XRD analysis. Although

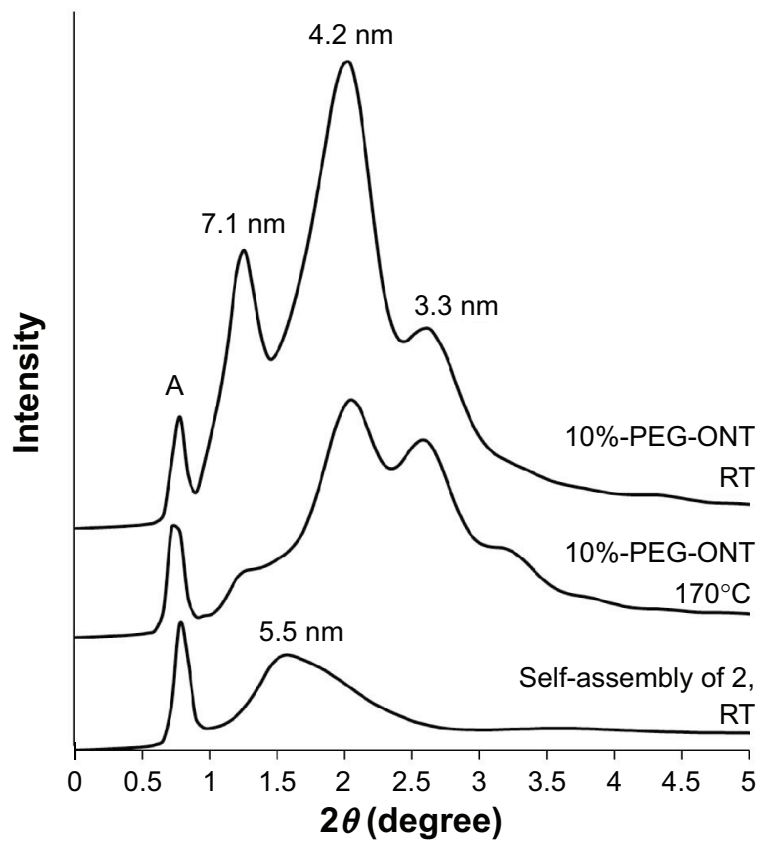

Figure 8 The XRD pattern of $10 \%$ PEG-ONT at RT and $170^{\circ} \mathrm{C}$, and self-assembly of 2 at RT.

Notes: The data on the peaks means the measured $d$-spacing values. Peaks $A$ (artifact) stem from the edge of beam stopper.

Abbreviations: ONT, organic nanotube; PEG, polyethylene glycol; RT, room temperature; XRD, X-ray diffraction.

the reason why the transition became exothermic was unclear, the possible detachment of $\mathbf{2}$ might induce subsequent rearrangement into a highly ordered molecular packing within the nanotubes, leading to an exothermic event.

To clarify the molecular packing of PEGylated ONTs and confirm the phenomenon of heat-induced phase separation, lyophilized $10 \%$ PEG-ONT and self-assembly of $\mathbf{2}$ were examined by XRD analysis (Figure 8). Ten percent PEG-ONT showed three diffraction peaks with $d$-spacing values of $7.1 \mathrm{~nm}, 4.2 \mathrm{~nm}$, and $3.3 \mathrm{~nm}$ (a weak shoulder), while self-assembly of $\mathbf{2}$ only gave a broad peak around $5.5 \mathrm{~nm}$. Compared to the previously reported mother ONT showing a single $d$-spacing of $4.02 \mathrm{~nm} ;{ }^{15}$ the main $d$-spacing peak of $4.2 \mathrm{~nm}$ and shoulder peak of $3.3 \mathrm{~nm}$ were assigned to the two tilt patterns of $\mathbf{1}$ in $10 \%$ PEG-ONT. A different preparation method probably induced the slight difference of molecular arrangement in the nanotube, since the previous ONT was prepared by cooling of hot DMSO solution and the latter PEGylated ONTs by $\mathrm{pH}$ adjustment method in 2-propanol- $\mathrm{H}_{2} \mathrm{O}$ solution.

It is remarkable that the sharp peak of $7.1 \mathrm{~nm}$ was only found in the $10 \%$ PEG-ONT. Such a large $d$-spacing value might indicate the large $\mathrm{PEG}_{2000}$ chain coil is exposed onto the outer surface, although we did not observe the PEG chains on electron microscopy. To some extent, this 
speculation was supported from the thickness of $\mathrm{PEG}_{2000}$ layer observed on liposomes. Based on small angle X-ray scattering study, the thickness of the $\mathrm{PEG}_{2000}$ layer on liposomes was estimated to be $\sim 5 \mathrm{~nm}$ when the $\mathrm{PEG}_{2000}$ fraction is $10 \mathrm{~mol} \%{ }^{34}$ The self-assembly of $\mathbf{2}$ gave a broad peak with $d$-spacing of $5.5 \mathrm{~nm}$ as measured by the present powder XRD analysis. The difference in thickness of each PEG moiety was attributable to difference in density of PEGylation and morphology, which may cause deformation of random coils of PEG.

When the temperature of $10 \%$ PEG-ONT was raised to $170^{\circ} \mathrm{C}$, which is beyond the exothermic peak $\left(148^{\circ} \mathrm{C}\right)$, the $d$-spacings of $\mathbf{1}(4.2,3.3 \mathrm{~nm})$ were essentially unchanged. Obvious change was found for the $d$-spacing of the $\mathrm{PEG}_{2000}$ chain; the sharp peak of $7.1 \mathrm{~nm}$ almost disappeared into a small shoulder peak similar to that of self-assembly of $\mathbf{2}$. These results suggested that, during the transition, PEG-lipid 2 might dissociate from the ONTs, and subsequent rearrangement of 1 probably induced the exothermic transition peaks. Unlike $10 \%$ and 20\% PEG-ONT, the mother ONT and 5\% PEG-ONT did not show such phase separation at least up to $180^{\circ} \mathrm{C}$, indicating that the high fraction of PEGylation decreased the thermal stability of ONTs.

In conclusion, PEG-lipid 2 was completely incorporated into ONTs until a fraction of $10 \mathrm{~mol} \%$, and the $10 \%$ PEGONT showed excellent thermal stability both in aqueous dispersion and dehydrate conditions. In the dehydrated condition, a heat-induced phase separation of $\mathbf{2}$ from dry PEGylated ONTs was observed above $120^{\circ} \mathrm{C}$ on the first heating cycle.

\section{In vivo distribution}

Formulations containing DOTA-gadolinium complex have been widely employed as MRI contrast agents as well as tracers for the in vivo quantification of drug nanocarriers..$^{27,35,36} \mathrm{In}$ this study, $10 \mathrm{~mol} \%$ of DOTA-lipid 3 was coassembled with $\mathbf{1}$ and $\mathbf{2}$ and the ONTs were labeled with gadolinium(III) acetate (0.9 equivalent to $\mathbf{3})$ for the in vivo quantitative detection of ONTs by ICP-AES. Since dialysis treatment has removed any free $\mathbf{3}$, gadolinium ions should specifically complex with the ONTs on the inner surface. After the addition of 0.9 equivalent gadolinium(III) acetate into ONTs, the mixture was again subjected to dialysis to check the existence of any free gadolinium ions. The gadolinium ions in the dialysis solution were below the detection limit. This result suggested the successful coassembly of 3 into ONTs and labeling of ONTs with gadolinium. The concentration of gadolinium in the ONTs was about
$0.584 \mu \mathrm{mol} / \mathrm{mL}$, and the injected dose of gadolinium is calculated as $4.33 \mu \mathrm{mol} / \mathrm{kg}$ for each mouse. Such a dose might be too low to visualize the distribution of ONTs in all the subject organs by MRI. MRI contrast agents, such as liposomes and micelles, usually require high doses of gadolinium (such as higher than $30 \mu \mathrm{mol} / \mathrm{kg}$ ) to generate enough signal-to-noise ratio. ${ }^{27,37,38}$ Therefore, the alternative method ICP-AES was employed for qualifying the amount of ONTs in mouse tissues.

The PEGylation significantly affected the tissue distribution of ONTs and improved the persistence time in the blood circulation in mice (Figure 9). For DOTA-ONT at 1 hour after infusion, about $66 \%$ of injected dose was accumulated in the lung, 25\% in the liver, and less than 5\% in the blood. In contrast, highly dispersed PEGylated DOTA-ONT remarkably reduced the accumulation in the lung, increased the accumulation in the liver, and remarkably prolonged the circulation time in the blood. At 1 hour postinjection, about $30 \%$ of injected PEGylated DOTA-ONT remained in the blood. The amount in the blood was dropped to about $10 \%$ and less than $5 \%$ at 6 hours and 24 hours, respectively. The result of DOTA-ONT was quite similar to the lung accumulation of aforementioned poorly dispersed ONT, in which the high axial ratio morphology of ONT was accounted as one of the factors in the high lung accumulation. ${ }^{21}$ Drug nanocarriers larger than $6 \mu \mathrm{m}$ were widely acknowledged to be easily trapped by the lung capillaries via intravenous injection. ${ }^{39,40}$ By the same mechanism, DOTA-ONT specifically accumulated in the lung because of its agglomeration and size increase in the blood (Figure S2). We would like

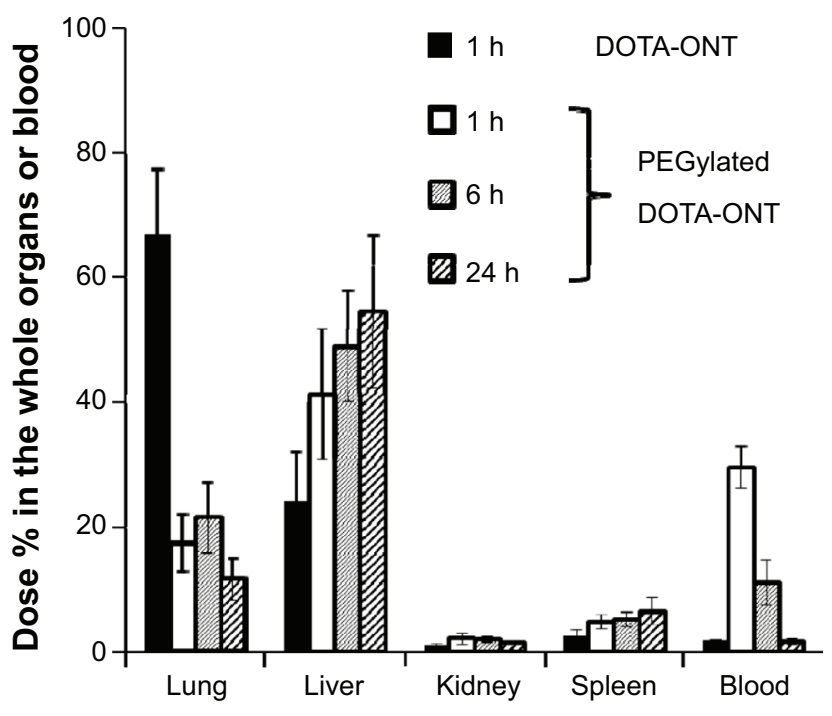

Figure 9 Distribution of DOTA-ONT and PEGylated DOTA-ONT in mice. Abbreviations: DOTA, tetraazacyclododecane tetraacetic acid; h, hour; ONT, organic nanotubes; PEG, polyethylene glycol; h, hour. 
to point out that such event was mainly responsible for the suffocation and instant death of mice after intravenous injection of DOTA-ONT. PEGylated DOTA-ONT avoided the accumulation in the lung simply due to its high dispersibility in biological medium, small size, and short length. In other words, PEGylation could also alleviate the acute toxicity of ONTs via intravenous injection.

Although PEGylated DOTA-ONT prolonged the circulation time in the blood, it still accumulated in the mouse liver; the persistence time was much like those of PEGylated carbon nanotubes, and they were much shorter than the polymeric filaments. ${ }^{4}$ A couple of studies reported that PEGylated carbon nanotubes also showed a circulation less than 24 hours. $^{26,41}$ Although the Young's modulus for polymeric filaments was not reported, self-assembled ONTs (Young's modulus $\mathrm{E} \sim 720 \mathrm{MPa})$ and carbon nanotubes $\left(\mathrm{E} \sim 10^{6} \mathrm{MPa}\right)$ were considered to have more high rigidness than the flexible filaments. ${ }^{42,43}$ Further, present PEGylated DOTA-ONT showed a mean length between 200 and $400 \mathrm{~nm}$, which may be too short to show "shape effect" as found in the long polymeric filaments (length is several micrometers). We suggest the high rigidness and short length are the main reasons for the lack of "shape effect" and its short blood persistence time in mice. Since long PEGylated ONT (in micrometer order) was difficult to prepare by the $\mathrm{pH}$-induced coassembly process, the effect of the aspect ratio on the in vivo distribution was not investigated.

Due to its biological stability and high aspect ratio, the existence of PEGylated ONT in the plasma was further visible under STEM observation (Figure 10). Many nanotubes were found in the plasma at 5 minutes after injection. The number of nanotubes decreased at 1 hour and 6 hours, and nanotubes seemed to be completely cleared from the blood after 24 hours. The direct visual STEM observation was quite coincide with the quantitative measurement of gadolinium by ICP-AES.

\section{Conclusion}

We successfully prepared PEGylated ONTs by a coassembly approach, and up to $10 \mathrm{~mol} \%$ PEG-lipid could be completely incorporated into ONTs. The PEGylation effectively prevented the aggregation of the ONTs in biological mediums. Because of the strong intermolecular interactions, heatinduced phase separation of PEG-lipid from dry PEGylated ONT was observed only when the temperature was higher than $120^{\circ} \mathrm{C}$, and no phase separation was observed for the $10 \%$ PEG-ONT dispersion under $95^{\circ} \mathrm{C}$. Compared to the nonPEGylated ONT, the PEGylation remarkably decreases the
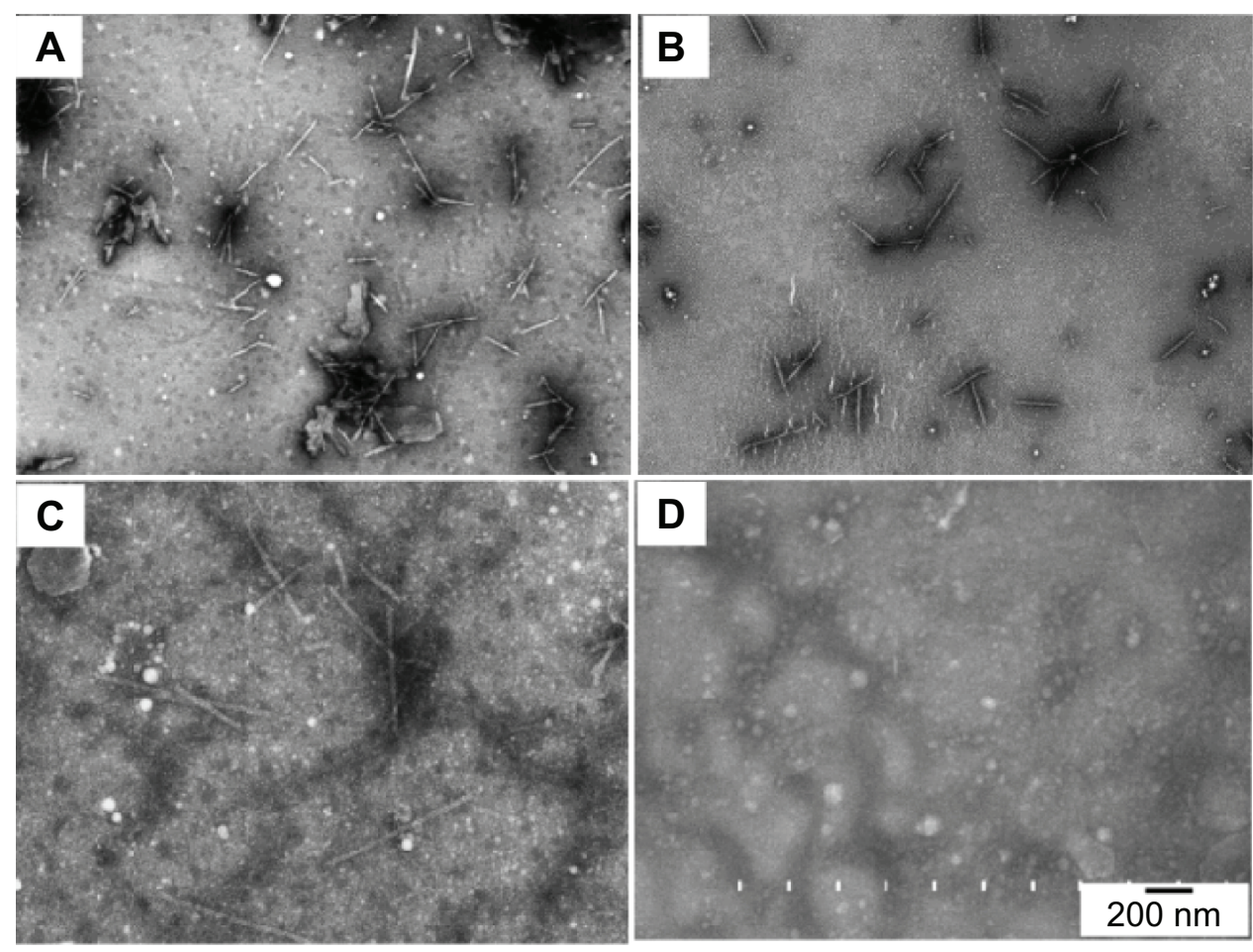

Figure 10 STEM images of plasma samples at (A) $5 \mathrm{~min},(\mathbf{B}) \mathrm{I} \mathrm{h},(\mathbf{C}) 6 \mathrm{~h}$, and (D) $24 \mathrm{~h}$ after the injection of PEGylated DOTA-ONT.

Abbreviations: DOTA, tetraazacyclododecane tetraacetic acid; h, hour; min, minutes; ONT, organic nanotube; PEG, polyethylene glycol; STEM, scanning transmission electron microscopy. 
accumulation of ONTs into the lung and improved the blood persistence time up to 24 hours in mice. Therefore, the PEGylated ONT holds promise as a novel drug nanocarrier because of its thermal stability and improved blood circulation.

\section{Acknowledgments}

The manuscript was written through contributions of all authors. All authors have given approval to the final version of the manuscript.

\section{Disclosure}

The authors report no conflicts of interest in this work.

\section{References}

1. Kostarelos K, Lacerda L, Pastorin G, et al. Cellular uptake of functionalized carbon nanotubes is independent of functional group and cell type. Nat Nanotechnol. 2007;2(2):108-113.

2. Gratton SE, Ropp PA, Pohlhaus PD, et al. The effect of particle design on cellular internalization pathways. Proc Natl Acad Sci U S A. 2008; 105(33):11613-11618.

3. Barua S, Yoo JW, Kolhar P, et al. Particle shape enhances specificity of antibody-displaying nanoparticles. Proc Natl Acad Sci U S A. 2013;110(9):3270-3275.

4. Geng Y, Dalhaimer P, Cai S, et al. Shape effects of filaments versus spherical particles in flow and drug delivery. Nat Nanotechnol. 2007; 2(4):249-255.

5. Yager P, Schoen PE. Formation of tubules by a polymerizable surfactant. Mol Cryst Liq Cryst. 1984;106(3-4):371-381.

6. Price R, Patchan M. Controlled release from cylindrical microstructures. J Microencapsul. 1991;8(3):301-306.

7. Schnur JM, Price R, Rudolph AS. Biologically engineered microstructures: controlled release applications. J Contr Rel. 1994;28(1-3): 3-13.

8. Spargo BJ, Cliff RO, Rollwagen FM, Rudolph AS. Controlled release of transforming growth factor-beta from lipid-based microcylinders. J Microencapsul. 2001;12(3):247-254.

9. MeilanderNJ, Yu X, Ziats NP, Bellamkonda RV.Lipid-based microtubular drug delivery vehicles. J Control Release. 2001;71(1):141-152.

10. Meilander NJ, Pasumarthy MK, Kowalczyk TH, et al. Sustained release of plasmid DNA using lipid microtubules and agarose hydrogel. $J$ Control Release. 2003;88(2):321-331.

11. Yan X, He Q, Wang K, et al. Transition of cationic dipeptide nanotubes into vesicles and oligonucleotide delivery. Angew Chem Int Ed Engl. 2007;46(14):2431-2434.

12. Wakasugi A, Asakawa M, Kogiso M, et al. Organic nanotubes for drug loading and cellular delivery. Int J Pharm. 2011;413(1-2):271-278.

13. Ding W, Wada M, Kameta N, et al. Functionalized organic nanotubes as tubular nonviral gene transfer vector. $J$ Control Release. 2011;156(1):70-75.

14. Song S, Chen Y, Yan Z, et al. Self-assembled rosette nanotubes for incorporating hydrophobic drugs in physiological environments. Int $J$ Nanomedicine. 2011;6:101-107.

15. Ding W, Kameta N, Minamikawa H, et al. Hybrid organic nanotubes with dual functionalities localized on cylindrical nanochannels control the release of doxorubicin. Adv Healthc Mater. 2012;1(6):699-706.

16. Ding W, Wada M, Minamikawa $\mathrm{H}$, et al. Cisplatin-encapsulated organic nanotubes by endo-complexation in the hollow cylinder. Chem Commun (Camb). 2012;48(69):8625-8627.

17. Kameta N, Lee SJ, Masuda M, Shimizu T. Biologically responsive, sustainable release from metallo-drug coordinated 1D nanostructures. J Mater Chem B. 2013;1(3):276-283.
18. Huang Z, Kang SK, Banno M, et al. Pulsating tubules from noncovalent macrocycles. Science. 2012;337(6101):1521-1526.

19. Hsieh WH, Chang SF, Chen HM, et al. Oral gene delivery with cyclo-(D-Trp-Tyr) peptide nanotubes. Mol Pharm. 2012;9(5): 1231-1249.

20. Panda JJ, Yandrapu S, Kadam RS, et al. Self-assembled phenylalanine$\alpha, \beta$-dehydrophenylalanine nanotubes for sustained intravitreal delivery of a multi-targeted tyrosine kinase inhibitor. $J$ Control Release. 2013;172(3):1151-1160.

21. Maitani Y, Nakamura Y, Kon M, et al. Higher lung accumulation of intravenously injected organic nanotubes. Int J Nanomedicine. 2013;8: 315-323.

22. Kogiso M, Zhou Y, Shimizu T. Instant preparation of self-assembled metal-complexed lipid. nanotubes that act as templates to produce metal oxide nanotubes. Adv Mater. 2007;19(2):242-246.

23. Kameta N, Yoshida K, Masuda M, Shimizu T. Supramolecular nanotube hydrogels: remarkable resistance effect of confined proteins to denaturants. Chem Mater. 2009;21(24):5892-5898.

24. Klibanov AL, Maruyama K, Torchilin VP, Huang L. Amphipathic polyethyleneglycols effectively prolong the circulation time of liposomes. FEBS Lett. 1990;268(1):235-237.

25. Gref R, Minamitake Y, Peracchia MT, et al. Biodegradable long-circulating polymeric nanospheres. Science. 1994;263(5153):1600-1603.

26. Liu Z, Davis C, Cai W, et al. Circulation and long-term fate of functionalized, biocompatible single-walled carbon nanotubes in mice probed by Raman spectroscopy. Proc Natl Acad Sci U S A. 2008; 105(5):1410-1415.

27. Shiraishi K, Kawano K, Minowa T, et al. Preparation and in vivo imaging of PEG-poly(L-lysine)-based polymeric micelle MRI contrast agents. J Control Release. 2009;136(1):14-20.

28. Baba $\mathrm{T}$, Zheng LQ, Minamikawa $\mathrm{H}$, Hato M. Interglycolipid Membrane Interactions: $\mathrm{pH}$-Dependent Aggregation of Nonionic Synthetic Glycolipid Vesicles. J Colloid Interface Sci. 2000;223(2): 235-243.

29. Takeoka S, Mori K, Ohkawa H, et al. Synthesis and assembly of poly(ethylene glycol)-lipids with mono-, di-, and tetraacyl chains and a poly (ethylene glycol) chain of various molecular weights. $J \mathrm{Am} \mathrm{Chem}$ Soc. 2000;122(33):7927-7935.

30. Baekmark TR, Pedersen S, Jørgensen K, Mouritsen OG. The effects of ethylene oxide containing lipopolymers and tri-block copolymers on lipid bilayers of dipalmitoylphosphatidylcholine. Biophys J. 1997;73(3): 1479-1491.

31. Hashizaki K, Taguchi H, Tsuchiya K, et al. Calorimetry and cryo-transmission electron microscopic studies of PEG2000-grafted liposomes. Chem Pharm Bull (Tokyo). 2006;54(4):561-563.

32. Pielichowski K, Flejtuch K. Differential scanning calorimetry studies on poly(ethylene glycol) with different molecular weights for thermal energy storage materials. Polym Adv Technol. 2002;13(10-12):690-696.

33. Allen TM, Hansen C, Martin F, et al. Liposomes containing synthetic lipid derivatives of poly(ethylene glycol) show prolonged circulation half-lives in vivo. Biochim Biophys Acta. 1991; 1066(1):29-36.

34. Varga Z, Wacha A, Vainio U, et al. Characterization of the PEG layer of sterically stabilized liposomes: a SAXS study. Chem Phys Lipids. 2012;165(4):387-392.

35. Le UM, Cui Z. Long-circulating gadolinium-encapsulated liposomes for potential application in tumor neutron capture therapy. Int J Pharm. 2006;312(1-2):105-112.

36. Huang $\mathrm{CH}$, Tsourkas A. Gd-based macromolecules and nanoparticles as magnetic resonance contrast agents for molecular imaging. Curr Top Med Chem. 2013;13(4):411-421.

37. Ye F, Ke T, Jeong EK, et al. Noninvasive visualization of in vivo drug delivery of poly(L-glutamic acid) using contrast-enhanced MRI. Mol Pharm. 2006;3(5):507-515.

38. Zhou Z, Lu ZR. Gadolinium-based contrast agents for magnetic resonance cancer imaging. Wiley Interdiscip Rev Nanomed Nanobiotechnol. 2013;5(1):1-18. 
39. Slack JD, Kanke M, Simmons GH, DeLuca PP. Acute hemodynamic effects and blood pool kinetics of polystyrene microspheres following intravenous administration. J Pharm Sci. 1981;70(6):660-664.

40. Kutscher HL, Chao P, Deshmukh M, et al. Threshold size for optimal passive pulmonary targeting and retention of rigid microparticles in rats. J Control Release. 2010;143(1):31-37.

41. Yang ST, Fernando KA, Liu JH, et al. Covalently PEGylated carbon nanotubes with stealth character in vivo. Small. 2008;4(7):940-944.
42. Frusawa H, Fukagawa A, Ikeda Y, et al. Aligning a single-lipid nanotube with moderate stiffness. Angew Chem Int Ed Engl. 2003; 42(1):72-74

43. Treacy MMJ, Ebbesen TW, Gibson JM. Exceptionally high Young's modulus observed for individual carbon nanotubes. Nature. 1996; 381:678-680. 


\section{Supplementary materials}

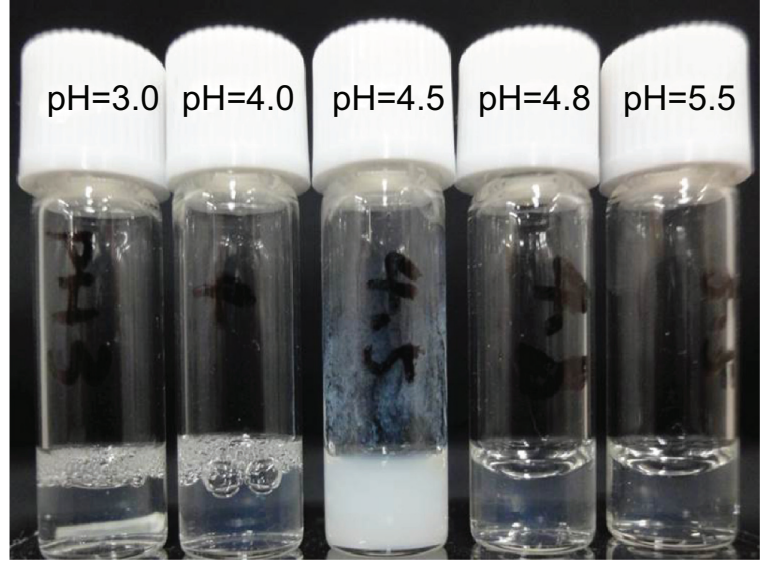

Figure SI pH-dependent self-assembly of DOTA-lipid 3.

Abbreviation: DOTA, tetraazacyclododecane tetraacetic acid.

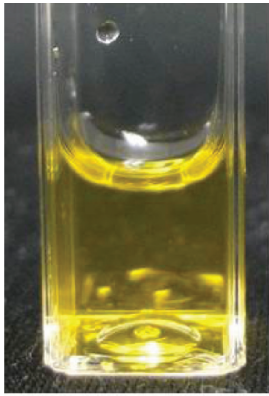

$1 \mathrm{~mL}$ plasma

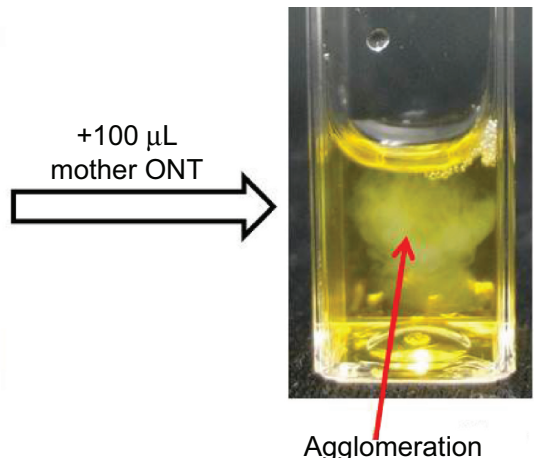

Agglomeration
Figure S2 Agglomeration of mother ONT in mouse plasma. Abbreviation: ONT, organic nanotube.

Table SI The exothermic temperatures and enthalpies of PEGylated ONTs and self-assembly of 2 based on DSC measurement

\begin{tabular}{lllll}
\hline Formulations & 5\% PEG-ONT & 10\% PEG-ONT & 20\% PEG-ONT & Self-assembly of 2 \\
\hline $\mathrm{T}\left({ }^{\circ} \mathrm{C}\right)$ of exothermic peak & $\mathrm{NF}$ & 148 & 129 & 103 \\
$\Delta H(\mathrm{~m} / \mathrm{mmol}$ of total lipid) & $\mathrm{NF}$ & -30.37 & -27.00 & $-58.5 \mathrm{I}$ \\
\hline
\end{tabular}

Abbreviations: DSC, differential scanning calorimetry; NF, not found; ONT, organic nanotube; PEG, polyethylene glycol.

A

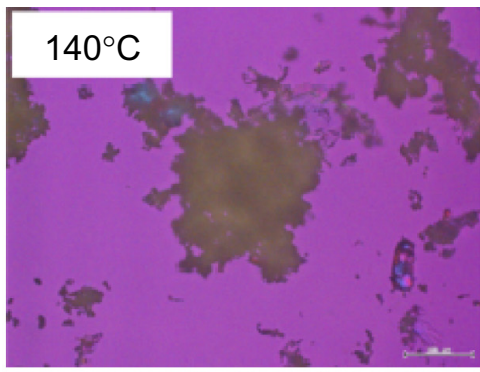

B

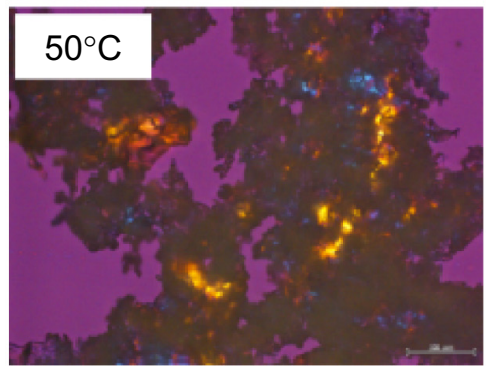

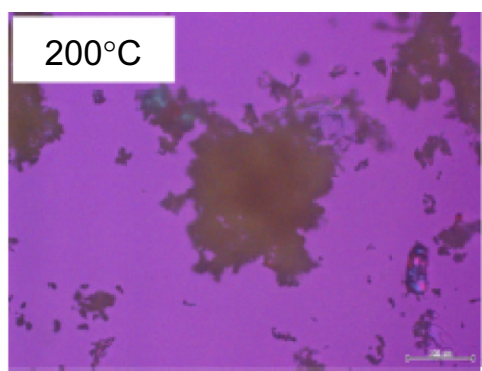
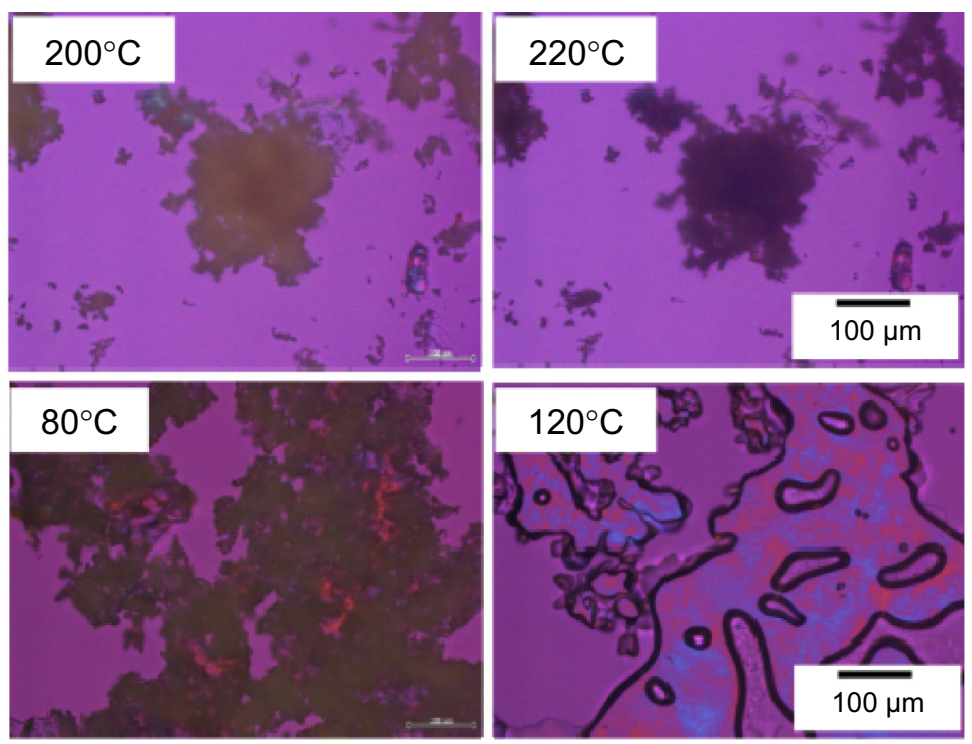

Figure S3 Optical microscopic images of (A) 10\% PEG-ONT, and (B) self-assembly of 2 at increased temperatures.

Notes: The self-assembly of 2 melted at $120^{\circ} \mathrm{C}$, while $10 \%$ PEG-ONT kept the solid state before decomposing (color turns black) at $220^{\circ} \mathrm{C}$.

Abbreviations: ONT, organic nanotube; PEG, polyethylene glycol. 


\section{Temperature $\left({ }^{\circ} \mathrm{C}\right)$}

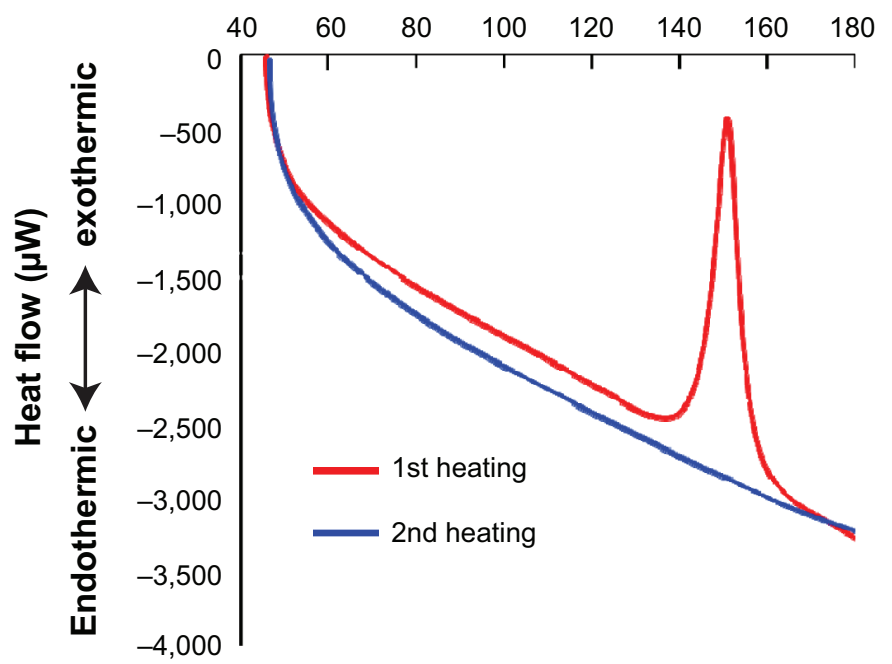

Figure S4 DSC cycles of dry 10\% PEG-ONT.

Abbreviations: DSC, differential scanning calorimetry; NF, not found; ONT, organic nanotube; PEG, polyethylene glycol.
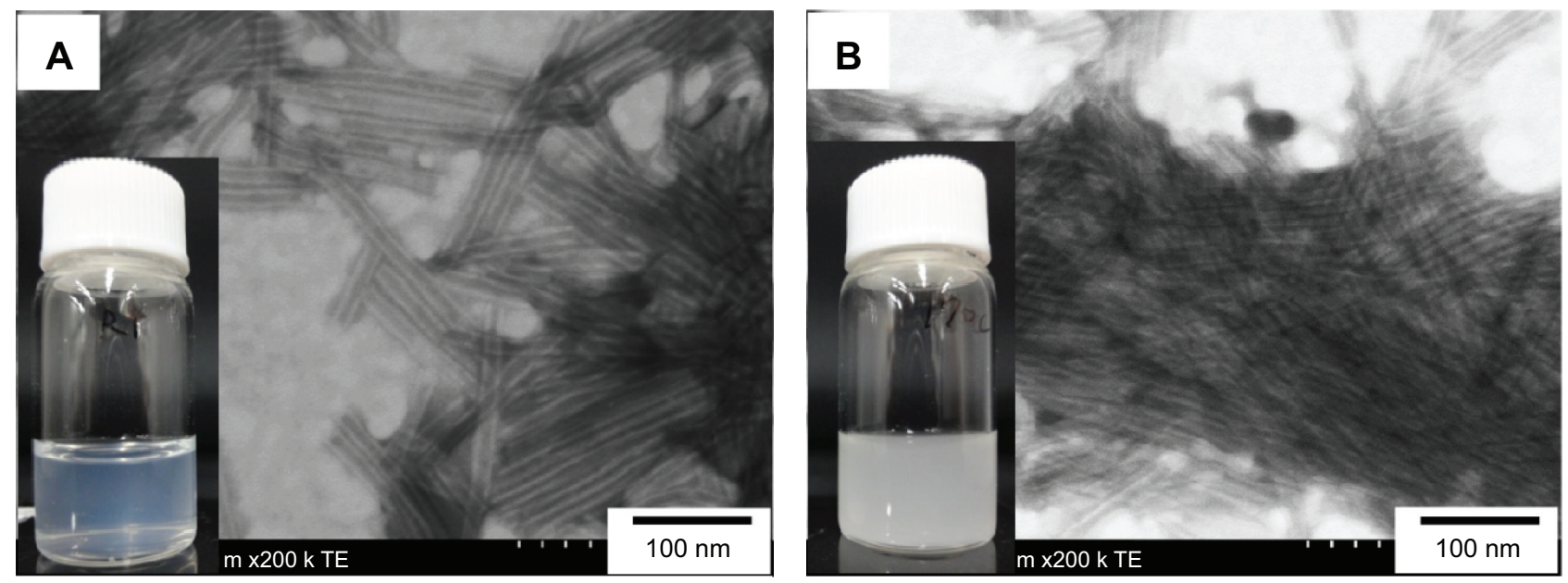

Figure S5 STEM images and macroscopic appearance (insets) of (A) nontreated and (B) heat-treated 10\% PEG-ONT in PBS buffer.

Notes: Freeze-dried 10\% PEG-ONT ( 6 mg) was untreated or treated at $170^{\circ} \mathrm{C}$ for $5 \mathrm{~min}, 5 \mathrm{~mL}$ of PBS buffer was added and the mixtures were sonicated for 5 min in a bath sonicator. Turbid dispersion indicated the phase separation of PEG-lipid 2 from 10\% PEG-ONT and nanotube aggregated in PBS buffer. Dissociation of 2 did not significantly destroy the nanotube structure.

Abbreviations: min, minutes; ONT, organic nanotube; PBS, phosphate-buffered saline; PEG, polyethylene glycol; STEM, scanning transmission electron microscopy.

International Journal of Nanomedicine

\section{Publish your work in this journal}

The International Journal of Nanomedicine is an international, peerreviewed journal focusing on the application of nanotechnology in diagnostics, therapeutics, and drug delivery systems throughou the biomedical field. This journal is indexed on PubMed Central, MedLine, CAS, SciSearch ${ }^{\circledR}$, Current Contents ${ }^{\circledR} /$ Clinical Medicine,

\section{Dovepress}

Journal Citation Reports/Science Edition, EMBase, Scopus and the Elsevier Bibliographic databases. The manuscript management system is completely online and includes a very quick and fair peer-review system, which is all easy to use. Visit http://www.dovepress.com/ testimonials.php to read real quotes from published authors.

\footnotetext{
Submit your manuscript here: http://www.dovepress.com/international-journal-of-nanomedicine-journal
} 